\title{
In Vitro Evaluation of Bond Strength to Primary Molar Dentine and Microleakage Properties of Low Polymerization Shrinkage Silorane Based Composite Resin
}

\author{
Buse Ayse Serin (Corresponding author) \\ Department of Pediatric Dentistry, \\ Faculty of Dentistry, Cukurova University, Adana, Turkey. \\ E-mail: dtbuse@hotmail.com \\ Iffet Yazicioglu \\ Department of Pediatric Dentistry, \\ Faculty of Dentistry, Cukurova University, Adana, Turkey. \\ E-mail: iffet_yazicioglu@yahoo.com \\ Muharrem Cem Dogan \\ Department of Pediatric Dentistry, \\ Faculty of Dentistry, Cukurova University, Adana, Turkey. \\ E-mail: cemdogan@cu.edu.tr
}

\begin{abstract}
Objective: The aim of this in vitro study is to evaluate the microleakage properties and shear bond strength to primary and permanent molar dentine of the low polymerization shrinkage silorane based composite resin and to compare the results with a methacrylate based composite resin. Materials and Methods: Shear bond strength test method was selected to evaluate the bond strength of the groups. Buccal dentine surfaces of primary and permanent molar teeth were used. The surfaces of the broken samples were detected under stereomicroscope and were grouped as adhesive, cohesive and mix. Dye penetration method was selected for the microleakage test. Class V cavities were prepared on the buccal surfaces of primary and permanent molar teeth. Dye penetration levels were recorded in accordance with determined scores.Results: Silorane based composite resin was showed lower shear bond strength for primary and permanent molar dentine $(\mathrm{p}<0.05)$. It was observed that most of the failures occurred in silorane based composite resin group, were adhesive type failures. According to the microleakage evaluation, primary molars were restored with silorane based composite resin were showed least microleakage $(p<0.05)$. Conclusion: The results of the laboratory tests should not be seen as the conclusion of the evaluated material but can be considered as preliminary information about the clinical performance of the material.
\end{abstract}

Keywords: Microleakage, Primary tooth, Shear bond strength, Silorane based composite resin.

\section{Special Issue of Health Sciences}

DOI: $10.7176 / J S T R / 6-03-14$

\begin{abstract}
1. Introduction
The use of composite resins in restorative dentistry become more popular with significant improvements has been made about their physical and mechanical properties. However polymerization shrinkage is still considered as the main drawback of composite resin. Polymerization shrinkage is depending on the intrinsic property of the resin matrix, the amount of filler, and the rate of the cure ${ }^{1}$. Upon curing, the single resin molecules move towards each other and are linked by chemical bonds to form a polymer network. This reaction leads to a significant volume contraction. Clinically, de-bonding, microleakage
\end{abstract}


through marginal gaps, postoperative sensitivity, secondary caries, enamel fracture and clinical failure of restorations could occur.

A new resin matrix was developed for dental composite to overcome the shrinkage problem, which is based on the silorane chemistry and named as silorane based composite resin. This chemical building differs from methacrylate monomer chemistry of the conventional methacrylate based composite resins. During the polymerization volumetric shrinkage of up to $\% 5$ occurs when radicals react with methacrylate group of the monomers to form a cross linked network resulting in a reduction of the intermolecular distance of the free monomer molecules ${ }^{2}$. In contrast to the linear-reactive groups of methacrylate, the ring-opening chemistry of the silorane starts with the cleavage and opening of the ring systems. This process gains space and counteracts the loss of volume which occurs in the subsequent step, when the chemical bonds are formed. In total, the ring-opening polymerization process yields a reduced volumetric shrinkage. The silorane ring-opening monomers provide for low polymerization shrinkage so the volumetric shrinkage of the silorane based composite resin is $<\% 1^{3}$. Silorane is obtained from the reaction of oxirane and siloxane molecules. The silorane-based composite resin (SBC) exhibited low polymerization shrinkage due to the ring-opening oxirane monomer and increased hydrophobicity due to the presence of the siloxane species ${ }^{4,5}$.

The aim of this in vitro study is to evaluate the microleakage properties and shear bond strength to primary molar dentine of the low polymerization shrinkage silorane based composite resin and to compare the results with a methacrylate based composite resin.

\section{Methods and Materials}

\subsection{Tooth selection}

A total of 160 intact human extracted primary and permanent molar teeth which were free from caries and had no other microscopic defect were used for this in vitro study. Extracted teeth were collected at the time of extraction and were cleaned with ultrasonic scalers for surface debridement. Then they were stored in distilled water which was periodically changed every week for not more than 3 months until they were used in the study. 80 primary and permanent teeth were selected for shear bond strength test and 80 were selected for microleakage assessment.

Schematic tables of the groups and restorative materials were shown at figure 1 and 2.

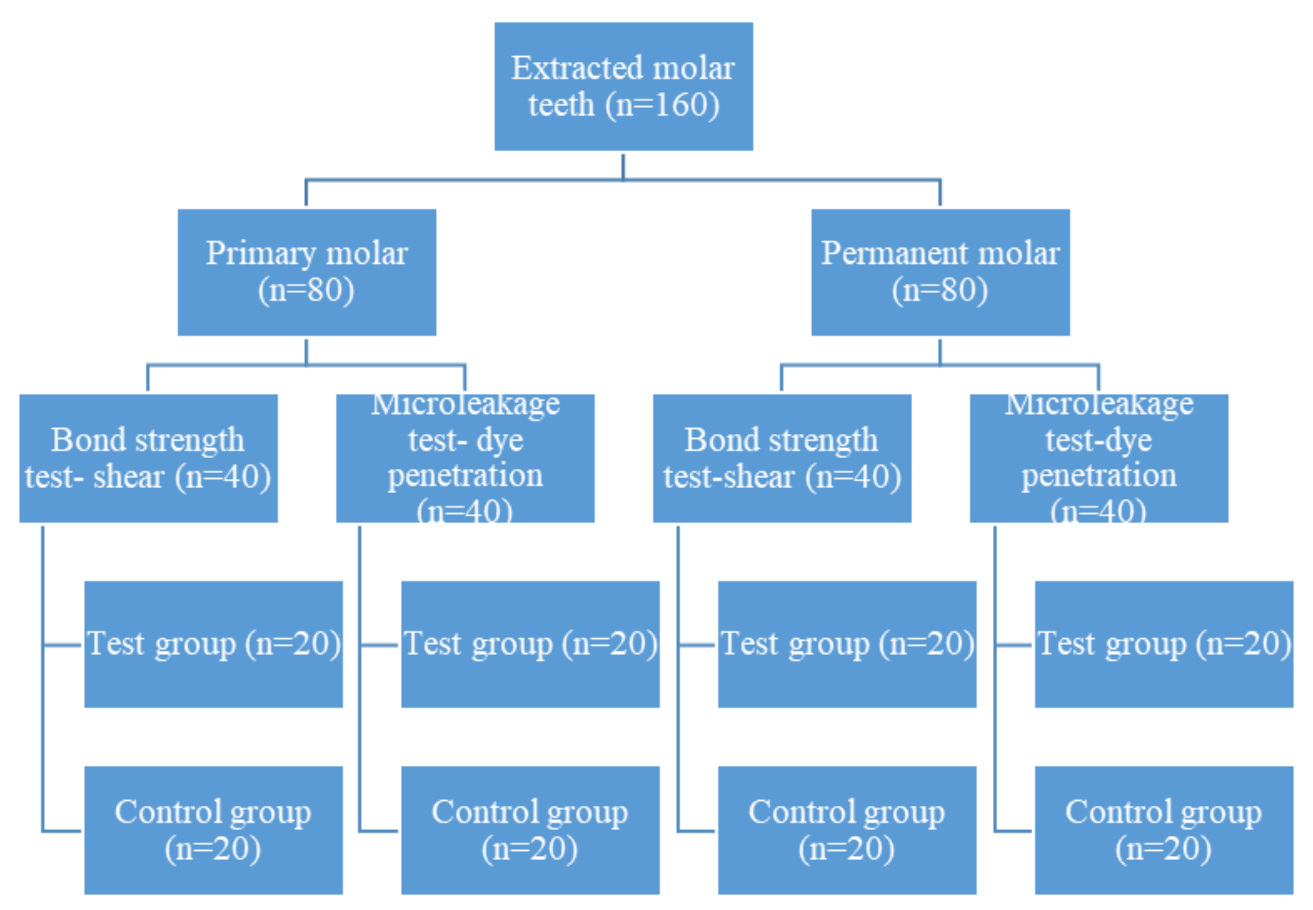

Figure 1: Groups. 

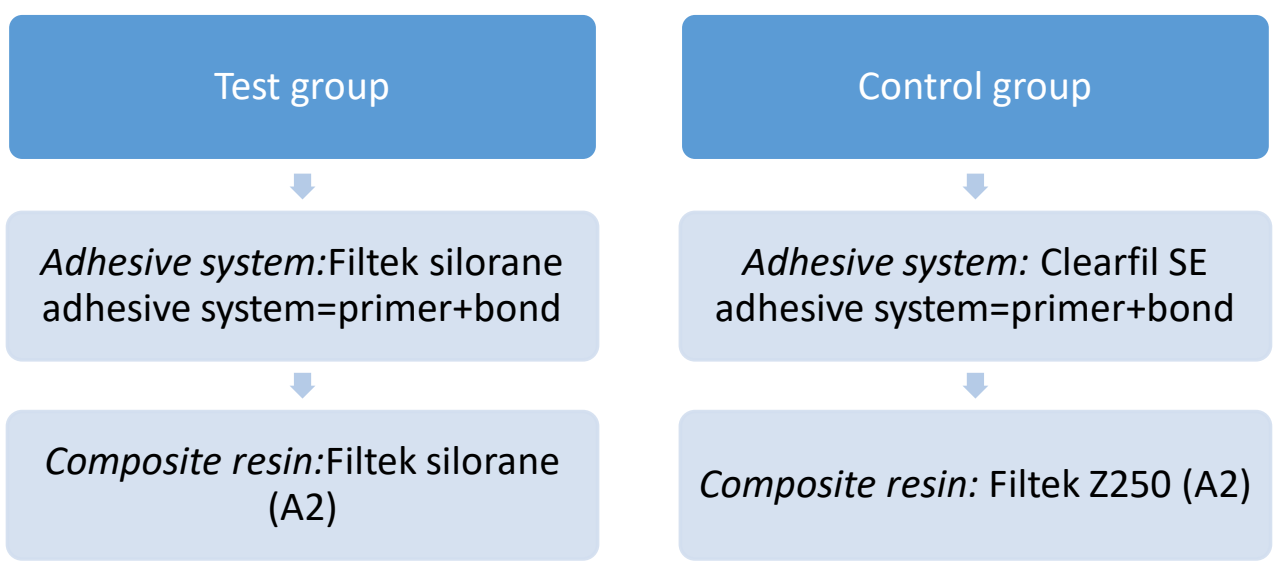

Figure 2: Restorative materials

\subsection{Methodology for bond strength test}

40 extracted non-carious primary molar teeth and 40 of the extracted non-carious permanent molar teeth were selected and divided into groups randomly.

Shear bond strength test method was selected to evaluate the bond strength of the groups. Roots were sectioned transversally with a water-cooled double-faced diamond disk, $2 \mathrm{~mm}$ below the cemento-enamel junction.

Buccal dentine surfaces of primary and permanent molar teeth were used. To remove the overlying enamel and expose a flat dentine surface, buccal dentine surfaces of teeth were prepared with round edge cylinder diamond bur (Meisinger Lot 527977 837LG012, Germany) by high speed hand piece with water-cooling. A bur was changed for every five teeth. 180 and 600 grit silicon carbide paper for 30 seconds was done in order to obtain a standard smear layer of dentine surfaces. After the mechanical preparation was completed, all buccal surfaces were examined under a stereomicroscope at a magnification of X25 (SZ 40, Olympus, Tokyo, Japan) to ensure the complete removal of enamel.

Stainless steel cylindrical molds were prepared in appropriate sizes (4 cm length, $2.7 \mathrm{~cm}$ diameter) as universal testing machine (Testometric Ax, M500-25kN, Rochdale, England). Teeth were embedded in these molds which were filled with autopolymerizing acrylic resin. Buccal surfaces of teeth were placed in the center of the mold and the dentin surface exposed in parallel to the ground plane. The samples were randomly divided into 4 groups $(n=20)$ according to test and control groups of primary and permanent teeth.

For the test group, the self-etch primer (Filtek Silorane System Adhesive, self-etch; 3M ESPE, St. Paul, USA) was applied to the surface for 15 seconds with a black microbrush, then air-dispersed carefully and light-cured for 10 seconds. The adhesive bond (Filtek Silorane System Adhesive, self-etch; 3M ESPE, St. Paul, USA) was then applied similarly with a green microbrush and light-cured for 10 seconds. SBC (Filtek Silorane low shrink posterior restorative; 3M ESPE, St. Paul, USA) in the shade of an A2 was applied incrementally into cylindrical shaped plastic matrix with a diameter of $4 \mathrm{~mm}$ and height of $5 \mathrm{~mm}$ by means of incremental technique, and then each increment was light cured with a light-emitting diode light-curing unit (Elipar Freelight; 3M ESPE, Seefeld, Germany) for 40 seconds.

For the control group, self-etch primer (Clearfil SE Bond primer; Kuraray Medical Inc, Okayama, Japan) was applied for 20 seconds and was dried thoroughly with mild air. Then adhesive bond (Clearfil SE Bond bond; Kuraray Medical Inc, Okayama, Japan) was applied, air-flowed gently and light-cured for 10 seconds. Methacrylate-based composite resin (MBC) (Filtek Z250; 3M ESPE,St Paul,MN, ABD) in the shape of A2 was applied into a cylindrical-plastic matrix with a diameter of $4 \mathrm{~mm}$ and height of 5 $\mathrm{mm}$ by means of an incremental technique and then was light-cured with a light-emitting diode lightcuring unit for 40 seconds.

Specimens were inserted into the slot with the help of a screw, fixed and then sheared with a ring blade on a universal test machine at a crosshead speed of $1.0 \mathrm{~mm} / \mathrm{min}$. The time of fracture was recorded in Newtons of force, next the shear bond strength was calculated in megapascals (MPa) by dividing the peak load at failure with the specimen surface area. (figure $3 a-b-c)$ 
The surfaces of the broken samples were detected under stereomicroscope and were grouped as adhesive, cohesive and mix.
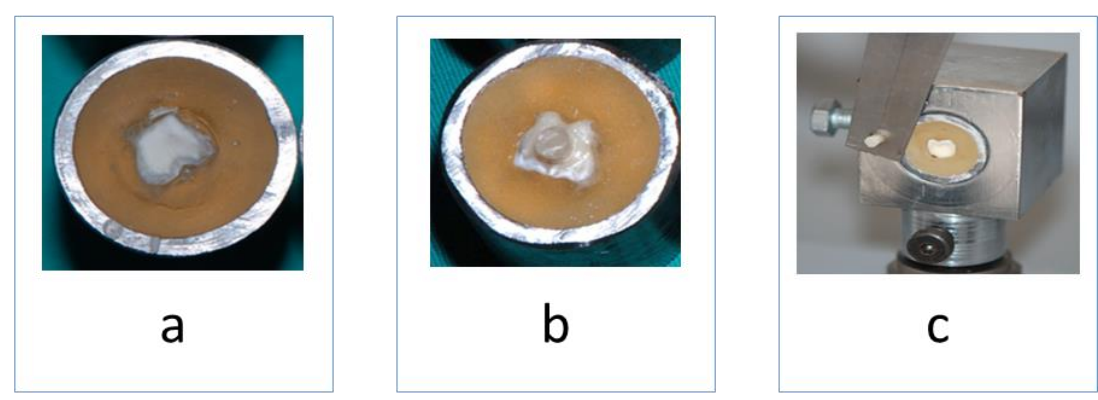

Figure3: a: a tooth was embedded in a stainless steel cylindrical mold which were filled with autopolymerizing acrylic resin. b: A tooth with composite resin which was applied into a cylindricalplastic matrix with a diameter of $4 \mathrm{~mm}$ and height of $5 \mathrm{~mm}$ by means of an incremental technique and then was light-cured. A specimen which was ready for shear bond strength test. c: The time of fracture.

\subsection{Methodology for microleakage test}

Eighty non carious extracted primary and permanent molars were collected dye penetration method was selected for the microleakage test. Class V cavities were prepared on the buccal surfaces of primary and permanent molar teeth, with the occlusal margins located $1 \mathrm{~mm}$ apical to the cement-enamel junction. The cavity dimensions were standardized with a template and controlled by a pre-marked periodontal probe that was $3 \mathrm{~mm}$ wide, $2 \mathrm{~mm}$ high and $2 \mathrm{~mm}$ depth.

The same materials and groups of the shear bond strength test method were used in the microleakage test. (figure 2) Thermal cycling was applied for 1000 times. Each cycle consisted of 30 seconds at $5^{\circ} \mathrm{C} \pm 2^{\circ} \mathrm{C}$ and 30 seconds at $55^{\circ} \mathrm{C} \pm 2^{\circ} \mathrm{C}$. The apices of the teeth were sealed with composite resin (Filtek $\mathrm{Z} 250$ ). The specimens were covered with two coats of nail varnish up to $1 \mathrm{~mm}$ from the restoration margin to prevent dye infiltration. (Figure 4) After keeping them in a $0.5 \%$ methylene blue solution for 24 hours, the superficial dye was removed by rinsed with distilled water and teeth were cut along the center line in the bucco-lingual direction through the midpoint of the restorations with a water-cooled, slow-speed diamond saw. Two sections of each tooth were obtained. The surfaces were examined under a stereomicroscope at $40 \mathrm{X}$ magnification by two examiners who were unaware of groupings and dye penetration levels were recorded in accordance with determined scores shown in table1 which the part of the tooth showed greater amount of dye penetration.

Table 1: Scores of microleakage

\begin{tabular}{|l|l|}
\hline Score & Definition \\
\hline 0 & No dye penetration \\
\hline 1 & Dye penetration up to $1 / 2$ of the gingival wall \\
\hline 2 & Dye penetration along the gingival wall \\
\hline 3 & Dye penetration up to $1 / 2$ of the axial wall \\
\hline 4 & Dye penetration along the axial wall \\
\hline
\end{tabular}

\subsection{Statistical analysis}

The results were recorded and analyzed using the statistical package Statistical Package for the Social Sciences (SPSS) 16.0 for Windows (SPSS Inc., Chicago,IL,USA.)

The comparison of the shear bond strength values between two different composite resin and primary molar teeth and permanent molar was carried out with 'One-Way ANOVA and post hoc Tukey's test. 
The difference between the means of the microleakage values of the anomaly distribution of the groups ' Kruskal Wallis test was used and comparisons between the groups was evaluated by Mann-Whitney U ' test.

Results at $95 \%$ confidence interval, significance at $\mathrm{p}<0.05$ is considered two-way.

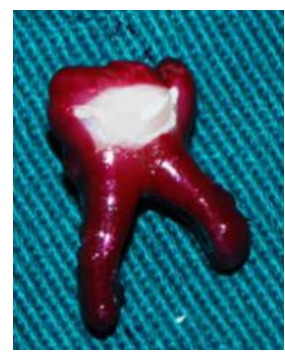

Figure 4: A specimen which was covered with two coats of nail varnish up to $1 \mathrm{~mm}$ from the restoration margin.

\section{Results}

\subsection{Shear bond strength}

The arithmetic mean and standard deviation values of shear bond values of all groups are shown in table 2. Silorane based composite resin was showed lower shear bond strength for primary and permanent tooth dentine $(\mathrm{p}<0.05)$. Primary tooth dentine were showed lower shear bond strengths for silorane based composite resin than permanent tooth dentine, whereas no significant difference was observed between primary and permanent tooth dentine for methacrylate based composite resin $(p<0.05)$.

Table 2: Shear bond strength of groups

\begin{tabular}{|c|c|}
\hline Grup & Mean \pm Sd (mpa) \\
\hline Primary tooth + Silorane & $18.7 \pm 0.8$ \\
\hline Primary tooth + Z250 & $23.6 \pm 2.91$ \\
\hline Permanent tooth + Silorane & $21.5 \pm 2.4$ \\
\hline Permanent tooth + Z250 & $25.8 \pm 1.9$ \\
\hline
\end{tabular}

The comparison of the difference of the average value of the shear bond strength of all groups are shown in table 3 (post hoc Tukey's multiple comparison test) (*statistically significant groups: $\mathrm{p}<0.05$ ).

After the shear bond strength test was performed fractured surface micromorphology was examined using a stereomicroscope (SZ 40; Olympus, Tokyo, Japan) at a magnification of X25. Specimen fractures were classified as follows: adhesive failure, cohesive failure or mixed failure when the two modes of failure happened. Fracture analysis was performed by a single observer who was completely uninformed about the experimental groups. 
Special Issue of Health Sciences, Vol.6, No.3, 2020

Table 3: The comparison of the difference of the average value of the shear bond strength of all groups (post hoc Tukey's multiple comparison test) (* Statistically significant groups: $\mathrm{p}<0.05$ )

\begin{tabular}{|c|c|c|c|}
\hline & Groups & Differences & Significance \\
\hline \multirow{2}{*}{$\begin{array}{c}\text { Primary tooth } \\
+ \text { Silorane }\end{array}$} & Primary tooth + Z250 & $-5.30703^{*}$ & .000 \\
& Permanent tooth + Z250 & $-6.59594^{*}$ & .000 \\
& Permanent tooth +Silorane & $-2.35667^{*}$ & .006 \\
\hline \multirow{2}{*}{$\begin{array}{c}\text { Primary tooth } \\
+ \text { Z250 }\end{array}$} & Primary tooth +Silorane & $5.30703^{*}$ & .000 \\
& Permanent tooth +Z250 & -1.28891 & .255 \\
& Permanent tooth +Silorane & $2.95036^{*}$ & .000 \\
\hline \multirow{2}{*}{$\begin{array}{c}\text { Permanent tooth } \\
+ \text { Silorane }\end{array}$} & Primary tooth +Silorane & $2.35667^{*}$ & .006 \\
& Primary tooth +Z250 & $-2.95036^{*}$ & .000 \\
& Permanent tooth +Z250 & $-4.23927^{*}$ & .000 \\
\hline \multirow{2}{*}{ Permanent tooth } & Primary tooth +Silorane & $6.59594^{*}$ & .000 \\
+ Z250 & Primary tooth +Z250 & 1.28891 & .255 \\
& Permanent tooth +Silorane & $4.23927^{*}$ & .000 \\
\hline
\end{tabular}

The fracture modes of the groups were presented in Table 4 and figure 5. It was observed that most of the failures occurred in silorane based composite resin group, were adhesive type failures.

Table 4: Fracture modes of groups

\begin{tabular}{|c|cc|cc|cc|cc|}
\hline Gruplar & \multicolumn{2}{|c|}{ Adhesive } & \multicolumn{2}{|c|}{ Cohesive } & \multicolumn{2}{|c|}{ Mixed } & \multicolumn{2}{|c|}{ Total } \\
& $\mathbf{n}$ & $(\boldsymbol{\%})$ & $\mathbf{n}$ & $\mathbf{( \% )}$ & $\mathbf{n}$ & $\mathbf{( \% )}$ & $\mathbf{n}$ & $(\boldsymbol{\%})$ \\
\hline $\begin{array}{c}\text { Primary tooth } \\
+ \text { Silorane }\end{array}$ & 15 & $(75)$ & 4 & $(20)$ & 1 & $(5)$ & 20 & 100 \\
\hline $\begin{array}{c}\text { Primary tooth } \\
+\mathbf{Z 2 5 0}\end{array}$ & 7 & $(35)$ & 5 & $(25)$ & 8 & $(40)$ & 20 & 100 \\
\hline $\begin{array}{c}\text { Permanent } \\
\text { tooth +Silorane }\end{array}$ & 12 & $(60)$ & 0 & $(0)$ & 8 & $(40)$ & 20 & 100 \\
\hline $\begin{array}{c}\text { Permanent } \\
\text { tooth +Z250 }\end{array}$ & 5 & $(25)$ & 5 & $(25)$ & 10 & $(50)$ & 20 & 100 \\
\hline
\end{tabular}




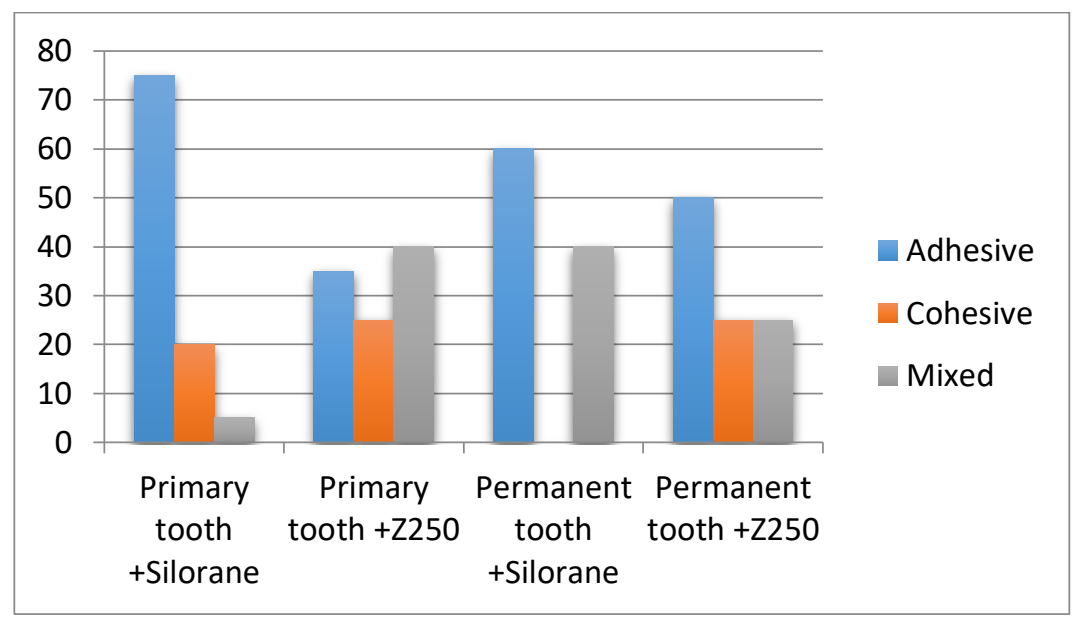

Figure 5: Column chart of fracture modes of the groups.

Table 5. Comparison of microleakage scores between groups

\begin{tabular}{|l|l|l|l|l|}
\hline Score & $\begin{array}{l}\text { Primary tooth } \\
+ \text { +Silorane } \\
(\mathbf{n = 2 0})\end{array}$ & $\begin{array}{l}\text { Permanent } \\
\text { tooth +Silorane } \\
(\mathbf{n = 2 0})\end{array}$ & $\begin{array}{l}\text { Primary tooth } \\
+\mathbf{Z 2 5 0}(\mathbf{n = 2 0})\end{array}$ & $\begin{array}{l}\text { Permanent } \\
\text { tooth }+\mathbf{Z 2 5 0} \\
(\mathbf{n = 2 0})\end{array}$ \\
\hline Score 0 & 4 & 5 & 0 & 0 \\
\hline Score 1 & 6 & 10 & 4 & 6 \\
\hline Score 2 & 9 & 5 & 6 & 5 \\
\hline Score 3 & 1 & 0 & 6 & 6 \\
\hline Score 4 & 0 & 0 & 4 & 3 \\
\hline Mean \pm sd & $1.5 \pm 0.9$ & $1 \pm 0.7$ & $2.5 \pm 1$ & $2 \pm 1$ \\
\hline Kruskal Wallis test & & \multicolumn{2}{|c|}{24.244} & \\
P value & & & $0.000^{*}$ & \\
\hline
\end{tabular}

$*$ According to Kruskall Wallis test, the groups were statistically different. $\mathrm{p}<0,05$

\subsection{Microleakage}

According to the microleakage evaluation, teeth were restored with silorane based composite resin were showed least microleakage $(\mathrm{p}<0.05)$. However, no different was found between primary and permanent teeth restored with the same composite resin $(p<0.05)$. Distribution of microleakage scores, mean and standard deviation values are shown in Table 5. And column chart of microleakage scores as percentange is in Figure 6.

Pair comparison of groups is shown in Table 6. When primary tooth + Silorane and Permanent tooth + Silorane groups were compared, it was seen that the mean microleakage score was less in the permanent tooth samples but this was not statistically significant.

When primary tooth + Silorane 'and primary tooth + Z250 groups were compared, the difference between microleakage scores was found to be statistically significant. According to this evaluation, primary-tooth + Silorane 'group showed less microleakage than primary-tooth + Z250' group. 
When Permanent tooth + Silorane and Permanent tooth + Z250 groups were compared, the difference between microleakage scores was found to be statistically significant. Permanent tooth + Silorane group showed less microleakage than the other group.

When primary tooth + Z250 and Permanent tooth + Z250 al groups were compared, it was seen that the mean mean score was lower in the permanent tooth samples but this was not statistically significant.

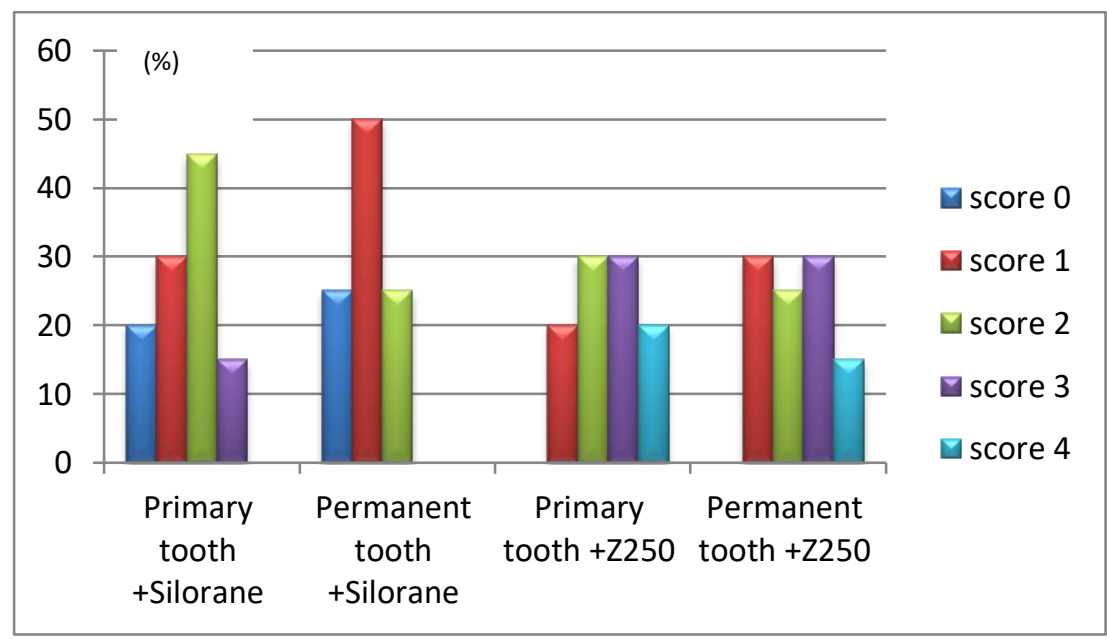

Figure 6. Column chart of microleakage scores of the groups.

Table 6. Pair comparison of groups according to Mann Whitney test.

\begin{tabular}{|c|c|c|c|}
\hline Groups & Mean \pm sd & $\mathbf{n}$ & $\mathbf{p}$ \\
\hline $\begin{array}{c}\text { Primary tooth } \\
\text { +Silorane }\end{array}$ & $1.5 \pm 0.9$ & 20 & \multirow[t]{2}{*}{0.171} \\
\hline $\begin{array}{l}\text { Permanent tooth } \\
+ \text { Silorane }\end{array}$ & $1 \pm 0.7$ & 20 & \\
\hline $\begin{array}{l}\text { Primary tooth } \\
\text { +Silorane }\end{array}$ & $1.5 \pm 0.9$ & 20 & \multirow[t]{2}{*}{$0.002 *$} \\
\hline Primary tooth $+\mathrm{Z250}$ & $2.5 \pm 1$ & 20 & \\
\hline $\begin{array}{c}\text { Permanent tooth } \\
\text { +Silorane }\end{array}$ & $1 \pm 0.7$ & 20 & \multirow[t]{2}{*}{$0.000 *$} \\
\hline $\begin{array}{l}\text { Permanent tooth } \\
+\mathrm{Z250}\end{array}$ & $2 \pm 1$ & 20 & \\
\hline $\begin{array}{l}\text { Primary tooth } \\
\text { +Silorane }\end{array}$ & $1.5 \pm 0.9$ & 20 & \multirow[t]{2}{*}{$0.010 *$} \\
\hline $\begin{array}{l}\text { Permanent tooth } \\
+\mathrm{Z250}\end{array}$ & $2 \pm 1$ & 20 & \\
\hline $\begin{array}{c}\text { Permanent tooth } \\
\text { +Silorane }\end{array}$ & $1 \pm 0.7$ & 20 & \multirow[t]{2}{*}{$0.000 *$} \\
\hline Primary tooth $+\mathrm{Z250}$ & $2.5 \pm 1$ & 20 & \\
\hline Primary tooth $+\mathrm{Z250}$ & $2.5 \pm 1$ & 20 & \multirow[t]{2}{*}{0.556} \\
\hline $\begin{array}{c}\text { Permanent tooth } \\
+\mathrm{Z250}\end{array}$ & $2 \pm 1$ & 20 & \\
\hline
\end{tabular}

\subsection{Images Taken Under Stereomicroscope}

Specimens determined as score 0 were not seen only in the primary + Silorane + and 'Permanent tooth + Silorane groups. The image of a sample whose microleakage value is determined to be 0 is given in figure 7.The samples with microleakage score of 1 were mostly found to be in the 'Permanent tooth + Silorane group however were seen in each group (Figure 8).

Microleakage score 2 although it was mostly seen in the 'primary tooth + Silorane', it was seen in each 
group (Figure 9).

The samples with microleakage score of 3 were seen in primary tooth + Z250 'and Permanent tooth + Z250 group but not in Permanent tooth + Silorane group (figure 10).

Specimens identified as Scores 4, which have the highest microleakage, were seen in groups primary tooth + Z250 'and Permanent tooth + Z250. The image of a sample with a microleakage score of 4 is given in Figure 11.

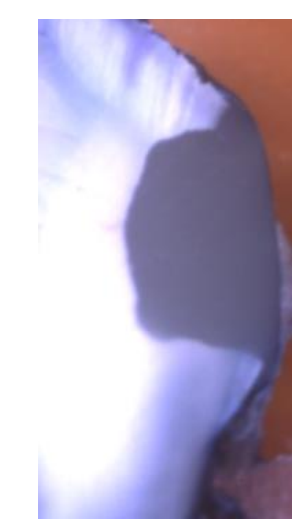

Figure 7. An image of a sample of the primary tooth + Silorane group under stereomicroscope with a score of $0 .(\mathrm{X} 100)$

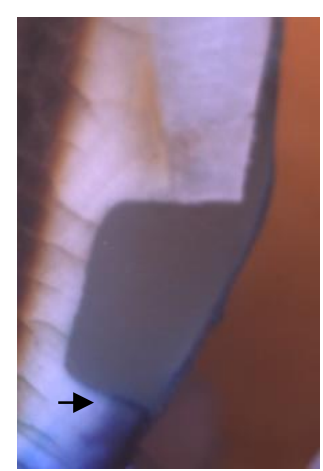

Figure 8. An image of a sample of the permanent tooth+ Silorane group under stereomicroscope with a score of 1. (X100)

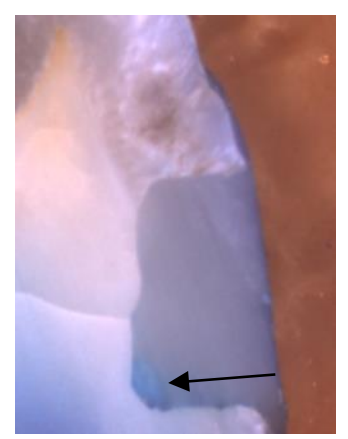

Figure 9. An image of a sample of the primary tooth + Silorane group under the stereomicroscope with a score of 2. (X100) 


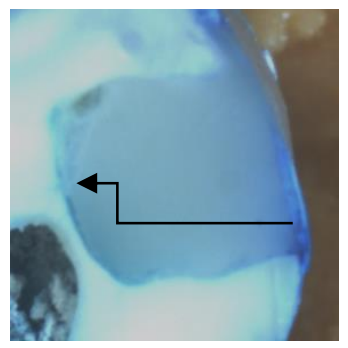

Figure 10. An image of a sample of the primary tooth $+\mathrm{Z} 250$ group under the stereomicroscope with a score of 3. (X100)

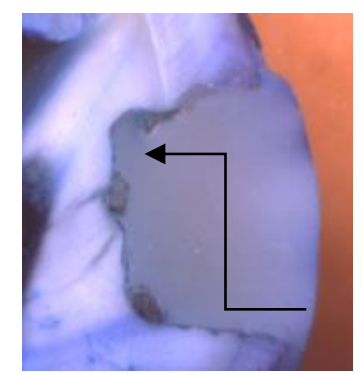

Figure 11. An image of a sample of the permanent tooth $+\mathrm{Z} 250$ group under the stereomicroscope with a score of 4. (X100)

\section{Discussion}

Restorative procedures for primary teeth should provide better marginal sealing, high bond strength and less technique sensitive. Composite resin restoration is a choice for restoration of primary teeth. However, there are some disadvantages of composite resin restorations according to polymerization shrinkage. To overcome these disadvantages alternative materials and techniques were developed. One of these, silorane based composite resin. This restorative material has been reported to result in significant reduction in polymerization shrinkage stresses and lower microleakage scores when compared to methacrylate based composite resins.

There are not presently available an ideal restorative material which provides a perfect seal with cavity walls. Several authors assume that gap size is positively correlated to microleakage values and attempts have been made to correlate bond strength values with marginal gap size ${ }^{6-9}$. Thus in this in vitro study we aimed to determine and compare microleakage and bond strength of silorane based composite resin with methacrylate based composite resin in both primary and permanent teeth.

If a restorative material achieves adequate bond strength to dental tissues, both microleakage and clinical complaints might be decreased. The practical way to evaluate the adhesive properties of restorative materials is in vitro bonding tests. Different bond strength test methods could be used to evaluate the clinical performance of restorative and adhesive systems ${ }^{10}$. Shear, tensile, microshear or microtensile tests were used to evaluate the bond strength of a composite resin ${ }^{11-14}$. Shear bond strength test was preferred in this study since it is a reliable and practical method for experimental evaluation of various dental material in vitro ${ }^{15}$.

Two different self-etch systems were used in this study. A self-etch adhesive system (Clearfil SE bond) was preferred for control group versus silorane based composite resin's own adhesive self-etch system. And two different composite resins were used.

In this study, the shear bond strength values of the group in which the Clearfil SE adhesive and the methacrylate-based composite resin was used were higher than the test group. The adhesive system of the silorane-based restorative system is in the weak self-etch adhesive group due to its high ph. Clearfil SE Bond, on the other hand, is in the medium-strength self-etch adhesive group. We believe that one of the reasons for the high bonding value is the ph. Another reason may be the effect of the MDP found within the Clearfil SE Bond system. Additionally, both the primer and the bonding agent of the Silorane self-etch adhesive system are light-polymerized. Both phases being light-polymerized resulting in high levels of monomer may have negatively affected the bonding. 
After applying self-etching adhesive systems to the tooth surface composite resin materials, plastic tubes into the area intended to provide a standard, incremental technique, $2 \mathrm{~mm}$ was applied in two separate layers.

The most important reason for the failure of composite resin polymerization shrinkage of the restoration and postoperative sensitivity can be improved accordingly, edge discoloration, secondary caries, such as the result of pulpitis or loss of restoration. Numerous studies have been conducted to modify composite resin content and to improve application techniques to reduce polymerization shrinkage.

The changes in the organic structure of the silorane-based composite resin ensure that the polymerization shrinkage is less than $1 \%$. While polymerization in conventional methacrylate-based composite resins occur with radical addition reaction, polymerization in silorane-based composite resins occurs with cationic ring opening reaction. In such reactions, ring-opening monomers open and bond with each other. Thus there is a lower volume loss resulting in less shrinkage. On the other hand, Filtek Z250 is a methacrylate-based composite resin and in such composite resins, the monomers are butt spliced with the radical addition polymerization. The loss in volume that occurs during the butt splicing of the monomers results in the polymerization shrinkage.

In this study, the bonding values of the group in which the Clearfil SE adhesive and the methacrylatebased composite resin was used were higher than the test group. The adhesive system of the siloranebased restorative system is in the weak self-etch adhesive group due to its high ph. Clearfil SE Bond, on the other hand, is in the medium-strength self-etch adhesive group. We believe that one of the reasons for the high bonding value is the ph. Another reason may be the effect of the MDP found within the Clearfil SE Bond system. Additionally, both the primer and the bonding agent of the Silorane self-etch adhesive system are photo-polymerized. Both phases being photo-polymerized resulting in high levels of monomer may have negatively affected the bonding.

According to the results of our study, the silorane-based composite resin showed average bonding values of $18.7 \mathrm{mpa}$ to primary teeth dentine and $21.5 \mathrm{mpa}$ to permanent teeth dentine; whereas the methacrylatebased composite resin showed average bonding values of $23.6 \mathrm{mpa}$ to primary teeth dentine and $25.8 \mathrm{mpa}$ to permanent teeth dentine. For both primary teeth and permanent teeth, the silorane-based composite resins have resulted in significantly lower shear bond strength than methacrylate-based composite resins. There are studies in the literature that compare and evaluate the bonding strength of silorane-based composite resins with various composite resins and adhesive systems. While the bonding strength of the silorane-based composite resins have been found to be lower in some of these studies, some studies have found no difference between the groups that were compared.

The 'Filtek Silorane Product Catalogue' indicates that the shear bond strength to dentine is $21 \mathrm{mpa}^{16}$. In a study evaluating the micro-shear bond strength of silorane-based composite resins on permanent teeth dentine in Class-1 occlusal restorations, Z250 showed the highest bonding strength (54.19 $\pm 9.05 \mathrm{mpa})$ while Silorane showed the lowest bonding strength $(6.94 \pm 2.07 \mathrm{mpa})^{17}$. In another study, the siloranebased composite resin showed lower bonding strength to permanent teeth dentine compared to Filtek Z250 and TPH Spectrum, which are methacrylate-based composite resins ${ }^{18}$. In a study evaluating the effect of the $\mathrm{C}$ factor on bonding strength, the bonding strength of the silorane-based composite resin was found to be lower compared to methacrylate-based composite resins ${ }^{19}$.

Another reason why silorane-based composite resins result in lower values may be due to the filler's volumetric ratio of silorane-based composite resin (55\%) being lower than that of Filtek Z250 (80\%). Although less polymerization shrinkage may seem like a clinical advantage, it brings certain problems along with it. Lowering the degree of polymerization to reduce the shrinkage tension negatively affects the mechanical properties of the composite resin. As the polymerization degree is lowered, the quantity of inert residual monomers increase. These residual monomers can negatively affect the bonding. An ideal composite resin is expected to show low levels of shrinkage while forming high degrees of polymerization.

Primary teeth have prismless outer surface layer and some investigators reported that this outer layer creates a reduction in the mechanical properties of restorative materials ${ }^{20,21}$. In our study, the bonding strength of silorane-based composite resin to primary teeth dentine have been found to be significantly lower compared to permanent teeth dentine. However, as the bonding strength of methacrylate-based composite resin to primary teeth dentine was found to be lower than that of the bonding strength to permanent teeth dentine, this difference was not found to be statistically significant. When the literature is reviewed, it is seen that certain studies have found no difference in the bonding of composite resins and adhesive systems to primary and permanent teeth dentine surfaces, whereas some studies have found that bonding to primary teeth dentine surfaces are weaker. The morphological properties of primary teeth differ from those of permanent teeth. These anatomical differences necessitate that the restorative approaches be different from each other. The dentine tubules of primary teeth are wider than those of 
permanent teeth. Also, mineral quantities and distributions vary compared to permanent teeth ${ }^{22-24}$. In the acid roughening of etch \& rinse adhesives, deeper demineralization is encountered in the intertubular dentine, tubular lumen width increases, and the amount of intertubular dentin to be utilized for adhesion is reduced. Additionally, there have been increases in dentinal reactivity after acid roughening in some studies and it has been suggested that lower concentration of acids be used for lower durations ${ }^{25,26}$. However, due to isolation problems that can be experienced with children, it is important to complete the clinical procedures in a shorter time with less technical adhesives. When these factors are considering together, the use of the self-etch adhesives in the restoration of primary teeth may be preferable.

In the final stage of our study on the bonding strength, the types of fractures formed by applying shear force on the sample surfaces were evaluated. The resulting fracture surfaces were examined with a light microscope. Adhesive type fractures were predominantly seen in groups using silorane-based composite resin. Adhesive type fractures show that the chemical bonding between materials is weak. The low shear bonding strength of silorane-based composite resin groups sheds light on the predominance of adhesive type fractures. The control group restored with Clearfil SE bond and Filtek Z250 that had cohesive and mixed fracture types having high bonding values was an expected result.

Microleakage is identified as the transition of bacteria, liquids, molecules, and ions between the cavity walls and the restorative material ${ }^{27}$. Polymerization shrinkage, dentine bonding strength, differences between thermal expansion coefficients of teeth and restorative material, and functional occlusal forces may lead to microleakage by creating gaps in the joining surface between the restoration material and dental tissue. The most important parameter in the formation of microleakage is polymerization shrinkage. Therefore the bonding strength of adhesives to dentine must be able to withstand the tension of polymerization shrinkage $e^{28-31}$. The elasticity module, shrinkage and thermal expansion coefficients of composite resins are the determining factors for preventing microleakage $\mathrm{e}^{32-34}$.

Dental caries, commonly seen in children, are more affected by microleakage compared to other cavity types. Such cases are explained with reasons such as the lack of enamel thickness in the cervical region, histomorphologic structure variations such as differences in the number and orientation of the enamel prisms in this region, existence of continuous pocket fluid flow or moisture contamination that can develop due to various reasons ${ }^{32-34}$. In order to quantify the amount of microleakage in the restorations in our study, the teeth were sectioned in the buccolingual direction using 2-dimensional evaluation method after the samples were kept in a dye solution. The microleakage values of all samples were then determined under the stereomicroscope.

According to the results of our microleakage study; silorane-based composite resin showed less microleakage in both primary and permanent teeth compared to methacrylate-based composite resin. There was no difference between the amount of microleakage in milk and permanent teeth restored with the same composite resin groups. The low amount of polymerization shrinkage may explain the lower occurrence of microleakage compared to groups using methacrylate-based composite resin.

The silorane-based composite resin is more hydrophobic than the methacrylate-based composite resin due to the siloxane structure found within its structure. Thanks to this feature, there is a reduction in water absorption and thus the related corresponding phenomena ${ }^{35}$. The hydrophobicity feature of the siloranebased composite resin may have caused the amount of microleakage to be lower compared to that of the methacrylate-based composite resin.

While our study found that the shear bond strength of silorane-based composite resin was lower compared to that of methacrylate-based composite resin, the silorane-based composite resin was found to be superior to the methacrylate-based composite resin when microleakage amounts were considered. When the literature is reviewed, there are contradictory results regarding the relationship between bonding strength and the ability to prevent microleakage ${ }^{36-39}$. Some researchers have found no correlation between bonding strength and microleakage ${ }^{36-39}$. In contrast to these studies, there are also studies which state that high in vitro bonding strength would reduce microleakage ${ }^{39,40}$. According to a study by Retief et al., it has been stated that an approximate bonding strength of $20 \mathrm{mpa}$ measured in vitro would be able to prevent marginal leakage in class 5 cavities $^{40}$. However, there is no systematic study in the literature linking the microleakage and bond strength between methacrylate-based conventional composite resins and silorane-based composite resins.

The low polymerization shrinkage of the composite resin, sufficient amount of bonding to the dental tissues, and the minimal amount of microleakage can ensure that the restoration can be long-lasting. According to the results of our study; the silorane-based composite resin which had lower polymerization shrinkage showed a lower bonding to primary teeth dentine than the methacrylate-based composite resin, but was found to be more successful in the microleakage test. The results of the laboratory tests should not be seen as the conclusion of the evaluated material but can be considered as preliminary information about the clinical performance of the material. The relationship between laboratory tests and clinical 
performance is inevitable, and the laboratory tests should be planned to be continued with clinical followups.

\section{Conclusion}

In this study, shear bond strength and microleakage of silorane based composite resin to primary molar dentine was investigated in vitro. However, some factors as occlusal stress, salivary-blood contamination and the child's cooperation couldn't mimicked with in vitro studies, the results of this study should be supported by an in vivo study and clinical controlled study, which should be planned, to determine more accurate performance of the material.

\section{References}

1. Maan M. AlShaafi. Factors affecting polymerization of resin-based composites: A literature review. Saudi Dent J. 2017;29(2):48-58.

2. Tae-Yub Kwon, Jung-Yun Ha, Ju-Na Chun, Jun Sik Son, and Kyo-Han Kim. Effects of Prepolymerized Particle Size and Polymerization Kinetics on Volumetric Shrinkage of Dental Modeling Resins. BioMed Research International. 2014: Article ID 914739, 6 pages.

3. Malhotra N1, Kundabala M, Shashirashmi A. Strategies to overcome polymerization shrinkage--materials and techniques. A review. Dent Update. 2010 ;37(2):115-8, 120-2, 124 5 .

4. Weinmann W, Thalacker C, Guggenberger R. Siloranes in dental composites. Dent Mater. 2005;21:68-74. doi: 10.1016/j.dental.2004.10.007.

5. Ghulman MA. Effect of cavity configuration (C factor) on the marginal adaptation of lowshrinking composite: a comparative ex vivo study. Int J Dent. 2011;2011:159749. doi:10.1155/2011/159749.

6. Rekha CV, Varma B, Jayanthi. Comparative evaluation of tensile bond strength and microleakage of conventional glass ionomer cement, resin modified glass ionomer cement and compomer: An in vitro study. Contemp Clin Dent. 2012;3(3):282-7.

7. Diwanji A, Dhar V, Arora R, Madhusudan A, Rathore AS. Comparative evaluation of microleakage of three restorative glass ionomer cements: An in vitro study. J Nat Sci Biol Med. 2014;5(2):373-7.

8. Omidi BR, Naeini FF, Dehghan H, Tamiz P, Savadroodbari MM, Jabbarian R. Microleakage of an Enhanced Resin-Modified Glass Ionomer Restorative Material in Primary Molars. J Dent (Tehran). 2018;15(4):205-213.

9. Jia S, Chen D, Wang D, Bao X, Tian X. Comparing marginal microleakage of three different dental materials in veneer restoration using a stereomicroscope: an in vitro study. BDJ Open. 2017;3:16010. Published 2017 Jan 6. doi:10.1038/bdjopen.2016.10

10. Mitra SB, Wu D, Holmes BN. An application of nanotechnology inadvanced dental materials. J Am Dent Assoc, 2003; 134(10): 1382-1390.

11. Baum L, Philips RW, Lund MR. Textbook of Operative Dentistry.3rd.Ed, USA: W.B.Saunders Co; 1995.

12.Pinkham JR, Casamassimo PS, Fields HW, McTigue DJ, Nowak A. Pediatric Dentistry, infancy through adolescence. 4th Ed, St. Louis, Elsevier Inc. 2005: 325-374. 
13. Braga RR, Ballester RY, Ferracane JL. Factors involved in the development of polymerization shrinkage stress in resin-composites: a systematic review. Dent Mater, 2005; 21(10): 962-70.

14. Wilson EG, Mandradjieff $M$, Brindock T. Controversies in posterior composite resin restorations. Dent Clin North Am, 1990; 34(1): 27-44.

15. Hofmann N, Siebrecht C, Hugo B, Klaiber B. Influence of curing methods and materials on the marginal seal of Class V composite restorations in vitro. Oper Dent, 2003; 28: 160-167.

16. Filtek Silorane. Technical Product Profile. USA,3M ESPE; 2007.

17. Krajangta N, Srisawasdi S. Microtensile bond strength of silorane-based resin composite and its corresponding adhesive in Class I occlusal restorations. Am J Dent, 2011; 24(6): 346-53.

18. Cabrera E, Macorra JC. Microtensile bond strength distributions of three composite materials with different polymerization shrinkages bonded to dentin. J Adhes Dent, 2011;13(1):39-48.

19.El-Sahn NA, El-Kassas DW, El-Damanhoury HM, Fahmy OM, Gomaa H, Platt JA. Effect of C-factor on microtensile bond strengths of low-shrinkage composites. Oper Dent, 2011; 36(3): 281-92.

20.Ripa LW, Gwinnett AJ, Buonocore MG. The "prismless" outer layer of deciduous and permanent enamel. Arch Oral Biol. 1966 Jan;11(1):41-8.

21.Zhang YR, Du W, Zhou XD, Yu HY. Review of research on the mechanical properties of the human tooth. Int J Oral Sci. 2014;6(2):61-9.

22.De Menezes Oliveira MA, Torres CP, Gomes-Silva JM, Chinelatti MA, De Menezes FC, Palma-Dibb RG, Borsatto MC. Microstructure and Mineral Composition of Dental Enamel of Permanent and Deciduous Teeth. Microsc Res Tech. 2010 May;73 (5):572-7. doi: 10.1002/jemt.20796.

23. Marangos O, Misra A, Spencer P, Bohaty B, Katz JL. Physico-mechanical properties determination using microscale homotopic measurements: application to sound and cariesaffected primary tooth dentin. Acta Biomater. 2009;5(4):1338-1348. doi:10.1016/j.actbio.2008.10.023

24.Lo Giudice G, Cutroneo G, Centofanti A, et al. Dentin Morphology of Root Canal Surface: A Quantitative Evaluation Based on a Scanning Electronic Microscopy Study. Biomed Res Int. 2015;2015:164065. doi:10.1155/2015/164065

25. Nör JE, Feigal RJ, Dennison JB, Edwards CA. Dentin bonding: SEM comparison of dentin surface in primary and permanent teeth. Pediatr Dent,1997;19(4):246-52.

26. Ölmez A, Öztaş N, Başak F, Erdal S. Comparison of the resin-dentin interface in primary and permanent teeth. J Clin Pediatr Dent, 1998; 22: 293-298.

27. Taylor MJ, Lynch E. Microleakage. J Dent, 1992; 20: 3-10.

28. Ali AM, Hamouda IM, Ghazy MH, Abo-Madina MM. Immediate and delayed micro-tensile bond strength of different luting resin cements to different regional dentin. J Biomed Res. 2012;27(2):151-8. 
29. Guimarães GF, Marcelino E, Cesarino I, Vicente FB, Grandini CR, Simões RP. Minimization of polymerization shrinkage effects on composite resins by the control of irradiance during the photoactivation process. J Appl Oral Sci. 2018;26:e20170528.

30.Loguercio AD, Reis A, Schroeder M, Balducci I, Versluis A, Ballester RY. Polymerization shrinkage: effects of boundary conditions and filling technique of resin composite restorations. J Dent. 2004 Aug;32(6):459-70.

31. Serin BA, Dogan MC, Yoldas HO. Comparison of the Shear Bond Strength of Silorane-Based Composite Resin and Methacrylate Based Composite Resin to MTA. J Dent Res Dent Clin Dent Prospects. 2018;12(1):1-5.

32.Lokhande NA, Padmai AS, Rathore VP, Shingane S, Jayashankar DN, Sharma U. Effectiveness of flowable resin composite in reducing microleakage - an in vitro study. J Int Oral Health. 2014;6(3):111-4.

33. Scotti N, Comba A, Gambino A, et al. Microleakage at enamel and dentin margins with a bulk fills flowable resin. Eur J Dent. 2014;8(1):1-8.

34.De Souza G, Braga RR, Cesar PF, Lopes GC. Correlation between clinical performance and degree of conversion of resin cements: a literature review. J Appl Oral Sci. 2015;23(4):358 368. doi:10.1590/1678-775720140524.

35.Al-Boni R, Raja OM. Microleakage evaluation of silorane based composite versus methacrylate based composite. J Conserv Dent, 2010;13(3):152-5.

36. Heintze SD. Systematic reviews: I. The correlation between laboratory tests on marginal quality and bond strength. II. The correlation between marginal quality and clinical outcome. J Adhes Dent. 2007; 9 Suppl 1: 77-106.

37. Heintze SD, Blunck U, Göhring TN, Rousson V. Marginal adaptation in vitro and clinical outcome of Class V restorations. Dent Mater, 2009; 25(5): 605-20.

38. Cenci M, Demarco F, de Carvalho R. Class II composite resin restorations with two polymerization techniques: relationship between microtensile bond strength and marginal leakage. J Dent, 2005; 33(7): 603-10.

39. Fortin D, Swift EJ Jr, Denehy GE, Reinhardt JW. Bond strength and microleakage of current dentin adhesives. Dent Mater, 1994;10(4): 253-8.

40. Retief DH, Mandras RS, Russell CM. Shear bond strength required to prevent microleakage of the dentin/restoration interface. Am J Dent, 1994;7(1):44-6. 